\title{
2-Hydroxyethyl Methacrylate
}

National Cancer Institute

\section{Source}

National Cancer Institute. 2-Hydroxyethyl Methacrylate. NCI Thesaurus. Code C47791.

A hydroxyester compound and a resin monomer used in desensitizing dentin. By applying 2-hydroxyethyl methacrylate locally to sensitive teeth, sensitive areas in the teeth get sealed and block the dentinal tubules at the dentin surface from stimuli that cause pain. This prevents excitation of the tooth nerve and relieves pain caused by tooth hypersensitivity. 